PROBLEMS OF ENGINEERING CYBERNETICS AND ROBOTICS • $2021 \bullet$ Vol. 76, pp. 3-24

p-ISSN: 2738-7356; e-ISSN: 2738-7364

https://doi.org/10.7546/PECR.76.21.01

\title{
The Internet of Things: \\ Description, Applications, Development, Challenges
}

\author{
Victor Danev \\ Institute of Information and Communication Technologies \\ at the Bulgarian Academy of Sciences \\ Acad. Georgi Bonchev Str., bl. 2, 1113 Sofia, Bulgaria \\ Emails: victordanev@abv.bg
}

\begin{abstract}
This overview describes not only the nature and applications of the Internet of things (IoT) but also some challenges and open research issues. Some of the established working definitions are presented, and the state of the IoT system is shown, incl. its reference model is based on a multi-layered architecture. Different characteristics of IoT are described and the communication technologies that are most often used are compared. Various applications of IoT and some challenges to the development of IoT innovations are shown. Based on the analysis of IoT, guidelines for future development of research in the field, which form the development of technology, are summarized.
\end{abstract}

Keywords: Internet of things (IoT), Definitions, Applications, Developments, Challenges.

\section{Introduction}

The concept of the Internet for non-use was first used in 1999 by Kevin Ashton (Atlam, Walters \& Wills, 2018). From Ashton's point of view, the Internet of things has the potential to change the world in different ways like using the Internet. Traditionally, technology can also be seen as the next step in the operation of the Internet. In practice, IoT can provide a connection to almost all objects in the real world (Sharabov \& Tsochev, 2020), including communication and collaboration via the Internet (Borissova, Dimitrova, Dimitrov, 2020). 
Although there is no generally accepted definition of the Internet of things in the academic and professional community, one of the most widely accepted working definitions of the phenomenon was presented by the International Telecommunication Union in 2012. According to the Union, the Internet of things should be understood as: a global infrastructure of the information society that provides the opportunity to create advanced services by interconnecting (physical and virtual) things from the real world, based on existing and evolving interoperable information and communication technologies. (ITU, 2012).

An interesting overview of reaching a definition is provided by Madakam, Ramaswamy \& Tripathi (2015), according to which the definition of the Internet of things includes: "an open and comprehensive network of intelligent objects that can self-organize, share information, data, and resources, reactions and actions in situations and changes in the environment". Beyond their definition, the authors also provide a quick overview of the most popular definitions, including:

- The Internet of things can be perceived as a global network of connected physical objects, which allows connectivity of all things at any time and not just for everyone (Kosmatos, Tselikas \& Boucouvalas, 2011);

- The Internet of things can mean a global network that allows communication between person and person, person and things, and things and things, that can relate to anything in the real world, providing a unique identity to each person and each object (Aggarwal \& Lal Das, 2012).

Beyond the nuances in the stated formulations, most researchers in the field of the Internet of things reach a consensus that the term generally means a connection between people, computers, and real objects through the Internet. A key prerequisite for real-world connectivity is the presence of sensors that convert raw data from the physical world into digital signals, which are sent to a control center (Suresh, Parthasarathy \& Aswathy, 2014).

\section{Description of IoT}

The Internet of things is often perceived as a "new paradigm" that belongs to the next wave of technological innovations that promote integration between the physical world and the cyber world (Colakovi \& Hadžialic, 2018). In addition to high-tech services, technology also provides highly personalized services in which the user interacts with specific "things" from the physical world through various communication models (Kirchev et al., 2006).

Over the last decade, the Internet of things technology has been characterized by exponential growth. One of the main factors that favor this is the 
low cost of production of sensors, communication protocols, embedded systems, and hardware components (Pattar et al., 2018). The main reason and motivation for such a development, however, is undoubtedly the unlimited industrial application of the technology.

It is important to note that the Internet of things is based on the integration of different standards and key technologies with different capabilities in terms of sensory, connectivity, storage, and more. This necessitates the development of models for collecting data from different devices (Vodyaho et al., 2020). On the other hand, intensive use of these technologies requires research to establish the psychological and physical effects on consumers (Borissova et al., 2020; Tianxing et al., 2021).

The specificity of the Internet of things consists in the way it is realized. This includes multi-layered architecture and communication technologies, which will be considered in turn.

\section{Architecture of IoT}

Based on a review of various academic sources, it can generally be assumed that the Internet of things architecture is a multi-layered architecture based on services (SOA-based architecture). To facilitate the industry in terms of technology deployment in practice, in 2014 the International Telecommunication Union published a reference model of the Internet of things architecture developed by Cisco, which is presented in Fig. 1:

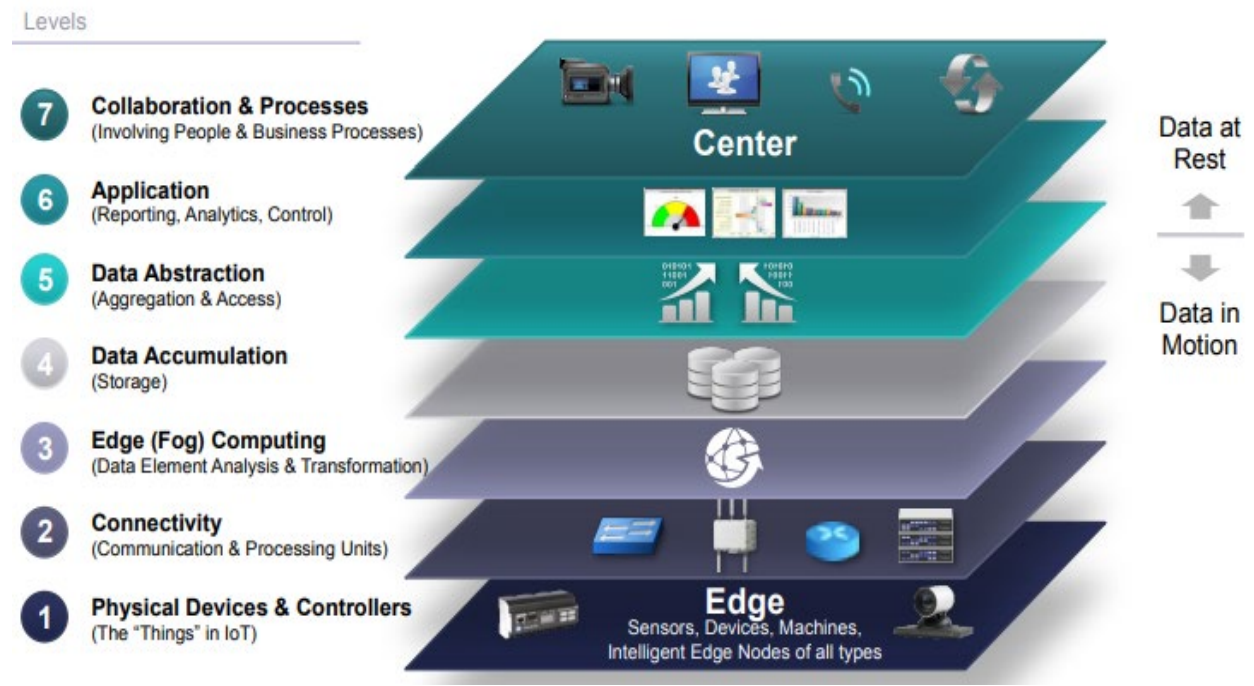

Fig. 1. Internet of things reference model [22] 
This model contains seven layers, each with its specific characteristics:

- Layer 1 - Physical devices and controllers. This layer represents the "things" in technology and consists of a huge number of different end devices that receive and send information. Although extremely different, these end devices are united by the following features: they can perform analog to digital conversion, generate data, and be controlled via the Internet.

- Layer 2 - Connectivity (communication network). The most important function of this layer is the transmission of information, which is realized at least at three levels: between the different devices and the network, between the different networks, between the network, and the processing of low-level information in layer 3.

- Layer 3-Peripheral calculations. This is the layer in which the primary processing of the information takes place so that it can be prepared for storage in layer 4 . The main processes that take place here include data evaluation, formatting, decoding, and more.

- Layer 4-Data collection. In this layer, the data is converted from a state of motion to a state of rest, stored, and prepared for use by higher levels.

- Layer 5-Data extraction. Here, the data is summarized and formatted so that it can be used by applications in a more efficient and manageable way.

- Layer 6-Applications. The interpretation of the data takes place in this layer. It includes several applications that can use data from the Internet of things as an input.

- Layer 7 -Cooperation and processes. In this last layer, the focus is on the people who work with the data and how the right data can reach the right people in the right way.

It can be assumed that this model, although derived from the industrial community, finds a good consensus in both business and academic circles.

\section{Features of IoT}

A brief and synthesized overview of the essential characteristics of the Internet of things is presented in (Atlam, Walters \& Wills, 2018). According to them, the Internet of things is characterized by the following features:

- large scale - refers to the significant number of included smart devices that generate data; 
- intelligence - with the help of software algorithms the devices included in the networks used in the Internet of things are transformed into smart devices;

- sensors - the Internet of things cannot be realized without sensors of objects in the physical world;

- complex systems - billions of devices can participate in the Internet of things;

- dynamic environment - in the system of the Internet of things objects are constantly included and excluded;

- huge amounts of data - this data is generated by the many devices involved in the system;

- heterogeneity - different devices, platforms, operating systems, and services participate in the Internet of things system, which connects through different protocols;

- minimum energy consumption - most of the devices on the Internet of things work with a minimum amount of energy;

- connectivity - devices on the Internet of things have the ability for network accessibility and compatibility;

- self-configuration - most devices are capable of self-configuration.

The IoT brings with it an exceptional range of benefits but also risks for the organizations that implement it. Some of them are related to big data management, which is both its advantage and challenge. The Internet of things can collect data from identification and tracking technologies, wired and wireless sensors, device networks, improved communication protocols, and other intelligent objects that are in constant data exchange. This also determines the immediate need to ensure their security and confidentiality (Fatkieva et al., 2020).

\section{Communication Technologies and IoT}

The IoT is a concept of the modern, interconnected world that relies on different heterogeneous communication technologies depending on the problem domain and quality of solutions based on the concept of IoT (Sikimic et al., 2020). The IoT comes with several high-end applications and has requirements for better connectivity through wireless communication protocols. The goal of IoT is to create an integrated ecosystem where devices can communicate with each other via the Internet network. This imposes to achieve an effective integration among Device to Device (D2D) communication technologies (Bello, Zeadally \& Badra, 2017). Low energy consumption is a prerequisite to ensure a continuous real-time 
connection of the transmitted data (Bahashwan et al., 2021). The heterogeneity of data originating from different devices is resolved through different communication protocols that allow devices to network and collaborate. Some of the main functions performed by the protocols are to specify the form of data exchange, to encode them, to control the sequence of exchange, and more. Communication protocols together with architecture form the backbone of the IoT system (Hofer-Schmitz \& Stojanovic, 2020).

The Internet of things uses many different communication protocols, the most common being ZigBee, Bluetooth, ZWave, 6LOWPAN, and NFC. Fig. 2 presents the wireless communication technologies used in the Internet of things, according to Colakovi \& Hadžialic (2018).

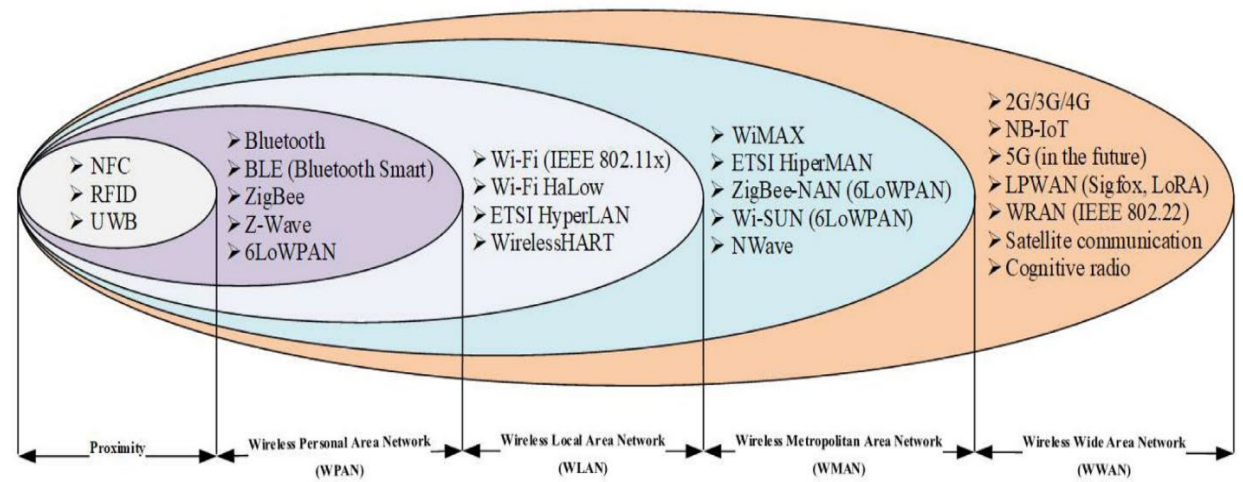

Fig. 2. Wireless communication technologies for IoT

Often, choosing wireless technology for the Internet of things is not a trivial task. Nevertheless, this choice can be determined both by the prevailing communication protocol, which is available in modern communication devices such as smartphones and by the purpose of the network (Taylor, 2019).

For completeness, this review will present the main features of some of the most commonly used communication technologies in the context of IoT.

\subsection{WiFi}

WiFi, along with Bluetooth, has been the most widely used communication protocol since 1997 . At present, the common $802.11 \mathrm{n}$ standard is widely used. It is based on IEEE 802.11 , but the application of 802.11 ac is also growing rapidly due to the ability to achieve low latency and high speed in the end networks (Hassani et al., 2021). Although this protocol is particularly convenient for communication between devices involved in the Internet of things, it uses a large 
amount of energy. Typically, WiFi is the preferred protocol for transferring files between devices (Taylor, 2019).

\subsection{ZigBee}

The ZigBee protocol was introduced by the ZigBee Alliance in 2004 and became operational in 2005. It is based on the open-source standard IEEE802.15.4, which has the lowest energy consumption. ZigBee is a suitable protocol for applications with a low data range, longer battery life, and better security (Balan et al., 2019). The protocol can work with a variety of technologies and is often used for automation solutions for home or industrial sites that operate on low energy consumption (Kuang \& Li, 2021).

\subsection{Bluetooth}

Bluetooth is also one of the most widespread communication protocols with the longest history and relatively short-range, usually up to 10 meters (Krasteva et al., 2005). Its more modern versions are called Bluetooth Smart or Bluetooth LowEnergy (BLE), and the latest version Ver. 5.0 technology has been developed and optimized specifically for the Internet of things (Todtenberg \& Kraemer, 2019). It also introduces an additional Internet Protocol file. The technology is preferred in smart wearables.

\subsection{EnOcean}

EnOcean technology is a pioneer in energy harvesting for sensors used by IoT (DeFeo, 2015). The technology allows the conversion of energy from the environment into digital data and is widely used on the Internet of things such as tracking maximum power and collecting photovoltaic energy (Ahmad et al., 2021).

\subsection{LoRaWAN}

The main application of the LoRaWAN protocol (Long Range Wide Area Network) is found in wireless devices with a wide range of operations that operate on batteries (Queralta et al., 2019). One of the strengths of this protocol is its ability to communicate widely. The protocol finds particularly good application in the construction of smart cities for air quality monitoring and due to communication technology (LoRaWAN) can be used in large geographical areas (Simo et al., 2021). 


\subsection{Z-Wave}

Z-Wave is a low-energy and radio-frequency communication protocol that provides reliable communication for small data packets developed by Zensys in 1999 (Tournier et al., 2020). The protocol operates with a frequency of the order of $900 \mathrm{MHz}$, which varies from country to country, and its range is in the diapason (30-100) $\mathrm{m}$, so it is assumed that its interaction with other communication protocols such as WiFi is negligible. It is used in the construction of smart home solutions (Kim et al., 2020).

\subsection{NFC}

The Near Field Communication (NFC protocol) provides simplified communication, connecting two devices that are less than $4 \mathrm{~cm}$ apart (Yugha \& Chithra, 2020). It is used for contactless payments, access to digital content, and connection to electronic devices.

\subsection{LoWPAN}

6LoWPAN is the first common Internet of things communication protocol (Low Power Wireless Personal Area Network IPv6). The protocol uses IEEE802.15.4 for its operation (Chalappuram et al., 2016). On this basis, it can be easily connected to other IP networks directly and without intermediaries.

All communication protocols discussed to support the Advanced Encryption Standard (AES) platform and, except for NFC, can be classified as a Wireless Personal Area Network (WPAN). NFC is a Peer-to-Peer (P2P) network.

\section{Applications of IoT}

Applications of IoT promise to bring immense value in lives due to newer wireless networks, modern sensors, and contemporary computing capabilities. The IoT applications in various industries and society as a whole are limitless. A general idea of the most popular industrial applications of the Internet of things is presented in Fig. 3 (Pattar et al., 2018).

The diagram covers mainly three main categories - industry, society, environment, which are deployed in different sectoral applications. Although schematic, this presentation is broad enough to illustrate the potential of the Internet of things to improve almost any area of the social and industrial world.

Of particular importance in recent years is the place of the Internet of things in the so-called "Industry 4.0", which is often seen as a new stage of the industrial revolution (Santhosh, Srinivsan \& Ragupathy, 2020). 


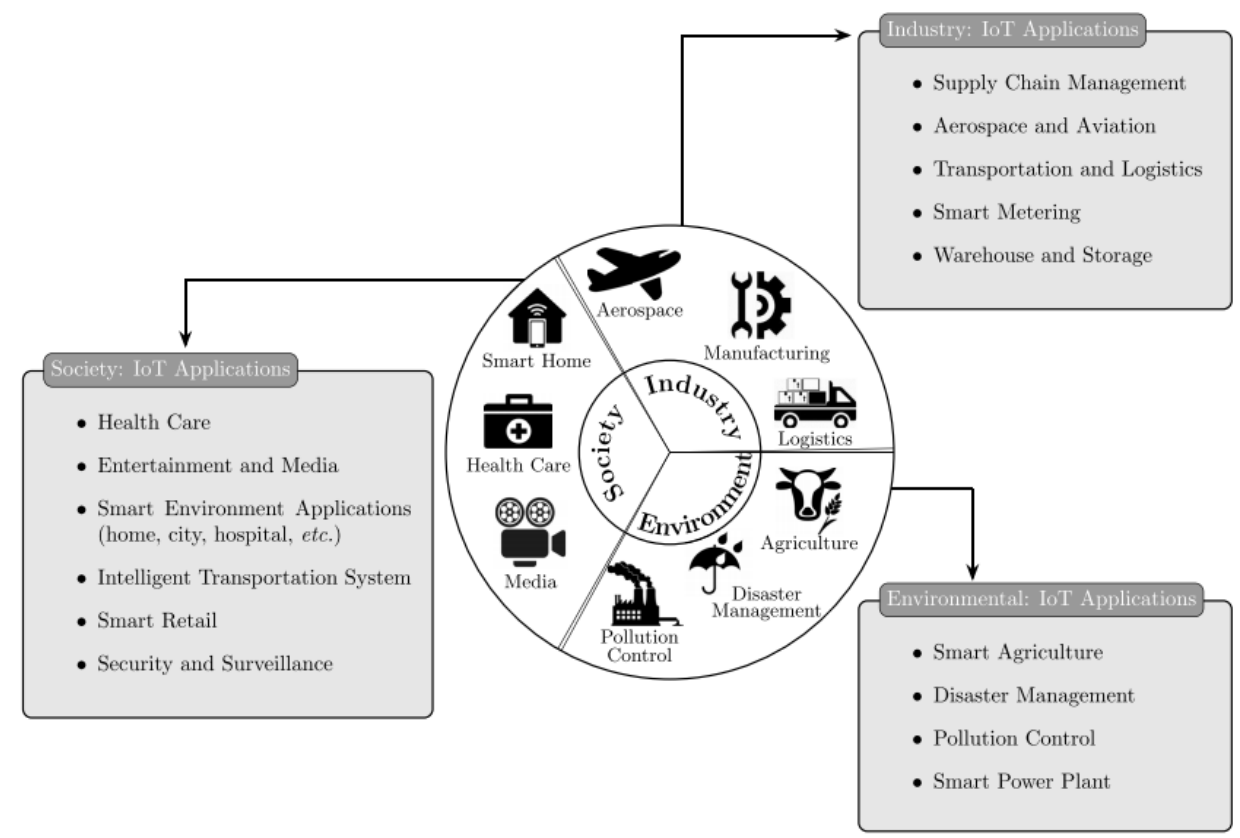

Fig. 3. Some of the most popular industrial applications of IoT

In this aspect, the literature usually discusses the application of the Internet of things in so-called smart factories. Their main prerequisite for work is the integration of all components of the existing industrial environment for better resource management through smart data management based on the information from sensors, software, and corresponding decision-making models (Mustakerov \& Borissova, 2013; Borissova \& Mustakerov, 2013; Fernandex-Carames \& Fraga-Lamas, 2018).

\subsection{Healthcare}

The contribution of the Internet of things to healthcare is undeniable. Three main phases can be distinguished such as clinical care, remote monitoring, and context awareness (Dhanvijay \& Patil, 2019). The phase related to data collection allows reducing the risks of human error using the automatic medical data collection method. This contributes to the improvement of the diagnosis quality and decreases the human errors responsible to collect or transmission of false information. The monitoring systems with sensors aim to collect and store a variety of data for further analysis (Gustafson et al., 2017). Context-awareness makes it possible to find the patient's condition (Vahdat-Nejad et al., 2021). 


\subsection{Smart cities}

The Internet of things is widely used in building smart cities with better services for citizens, lower costs, and more efficient management of public resources (Marques et al., 2019; Borissova et al., 2020). Areas such as urban transport management and other vehicles are essential for large settlements (Garvanov et al., 2021). Through the Internet of things, citizens have both better access to local public services and better access to information about their governance processes (Clement \& Crutzen, 2021).

\subsection{Smart homes}

The opportunity for people to facilitate the use of various innovative services in their homes has always enjoyed good development. The Internet of things gives additional impetus to these innovations by providing both remote control of devices that do not require the presence of the user to the home appliance and smart energy consumption, integration, safety, and many other benefits (Choi, Kim, Lee, Park, 2021). This technology is particularly important for facilitating the lifestyle of the elderly, people with disabilities, and other vulnerable groups (Debauche et al., 2019; Sokullu et al., 2020).

\subsection{Related industry}

This is one of the areas that is experiencing the most rapid industrial development. The focus is on the connectivity of different machines in enterprises, synchronization of their communication, productivity, with control and management capabilities to increase efficiency and for fair ethical business development (Margherita \& Braccini, 2021). Of particular importance is the opportunity provided by technology through the exchange of information and data so that machines can make their own decisions about implementation, automate processes, and more. (Santhosh, Srinivsan \& Ragupathy, 2020).

\subsection{Autonomous cars}

When cars are equipped with WiFi, through which they can communicate with other cars involved in traffic, the likelihood of human error, accidents, driving stress, and other similar risks are significantly reduced. An overview of the existing applications of FPGA for the design of control systems in autonomous cars is presented (Marinova, 2018). By 2040, 75\% of cars on the road are projected to be equipped with WiFi (Atlam, Walters \& Wills, 2018). 


\subsection{Intelligent energy-saving and Intelligent electricity networks}

The utmost importance attached to this sector by both governmental and international sustainable development programs stimulates research into the potential contribution of the Internet of things. Due to the complexity of the matter, it is considered that no single final solution has yet been found (Shah et al., 2018). Nevertheless, the new technology contributes to several improvements in energy saving, mainly through the introduction of smart energy management devices (Guliashki, Marinova \& Groumpos, 2019).

\subsection{Environmental monitoring}

The contribution of the Internet of things to environmental monitoring can be classified into a separate category. This is mainly due to the ability of the technology to deploy sensors over a large area, which extracts huge data on the various sources of environmental pollution and the quality of its various components such as land, air, water, biomass, etc. (Singh et al., 2021; Trevathan et al., 2021; Gopikumar et al., 2021). Data from such observations can be used by various stakeholders such as owners, managers, policy experts to facilitate forecasting, planning, and decision-making.

\subsection{Intelligent agriculture}

One of the most important aspects of the application of the Internet of things in agriculture is in helping farmers to control the parameters of the environment in the cultivation of production, as well as to improve production efficiency (Moysiadis et al., 2021; Boursianis et al., 2020). In addition, the technology can apply various aspects of automation to the entire production and logistics chain and thus facilitate the end-user with information about the origin and quality of the product (Huang et al., 2021; Carlos et al., 2020). The IoT application in agroindustrial and environmental fields is contributing to irrigation control, air, soil, and water monitoring including plant or crop monitoring as well as lumination and Fertilizer and pesticide control (Talavera et al., 2017).

\subsection{Wearables}

The main purpose of smart wearable devices is to collect, transmit and analyze data and in some cases to make intelligent decisions. Depending on their application, smart wearables are classified into four major groups: health, sports, and daily activity, tracking and localization, and safety (John Dian, Vahidnia $\&$ Rahmati, 2020)). The Internet of things can also be used for smart wearables to monitor the health status of quarantined patients with Covid-19 (Al Bassam et al., 
2021). In addition to improving the functionality of the modern accessories, we use in training, various smart devices introduce innovations to support the independent living of certain vulnerable groups. One example of this is smart devices that are designed to support the independent living of older people (Baig et al., 2018).

\section{Challenges}

As mentioned above, in addition to its many benefits, the Internet of things brings with it its challenges, the most important of which we have outlined in the section below.

\subsection{Big data}

The ability of the Internet of things to generate large amounts of data is directly related to the challenge of their integration, storage, and management. This is essential and forms the subject of analysis of several focused academic studies. For example, the dual effect of the Internet of things describing big data through the prism of the BOLD category (Big, Open, Linked Data) is presented in (Brous, Janssen \& Herder, 2020). According to the authors, through this concept, we can trace both the benefits and challenges of big data. One of the most obvious advantages of these data is their better quality compared to data generated by traditional means, especially in terms of accuracy, variety, speed of generation, and volume. Not to be underestimated is the ability of data being generated from one place to be used for different purposes. At the same time, however, their management faces the immediate challenges of infrastructure capacity, governance itself, ensuring their security and confidentiality (Tsochev et al., 2019). The latter aspects could hide other unforeseen risks, as well as lead to high costs for the organization when introducing the technology.

\subsection{Network provision}

Networking and the Internet of things system is a challenge itself, just as its network brings together both smart and traditional networks. The development of a network protocol and network typology creates related challenges that are not easy tasks (Aboubakar et al., 2021).

\subsection{Heterogeneity}

The wide variety of devices, operating systems, and services, as well as the constant expansion of networks that use the Internet of things, bring to the fore the problem of connectivity and coordination of different devices. Sometimes this 
heterogeneity can affect the design of the architecture and other aspects of the system (Mesmoudi et al., 2020). A related problem with heterogeneity is the standardization of things on the Internet, which is a major challenge for the future development of technology.

\subsection{Interoperability}

Interoperability refers to the ability of devices to communicate regardless of the hardware and software used. Given the interconnectedness of the various components and devices on the Internet of things, their interoperability is critical (Guimaraes et al., 2020). This is one of the areas that is developing quite rapidly due to market needs. Nevertheless, interoperability remains one of the open issues facing the development of networks that use the Internet of things.

\subsection{Scalability}

Scalability refers to the ability of a system to add new devices and services without affecting the technologies used. Thus, scalability related to the size of IoT systems becomes a significant concern (Arellanes \& Lau, 2020). Here the challenges are related to the maintenance of a large number of memory devices and other resources, directly affecting the interoperability and architecture design.

\subsection{Security and confidentiality}

Ensuring data security and confidentiality is currently a major challenge for the widespread use of the Internet of things (Babun et al., 2021). These two problems are directly related to big data management, but their guarantee is inevitable so that the user can enjoy the other benefits of the Internet of things.

The need for globalized IoT laws to ensure the common user to be aware of the security, ethical, and privacy threats due to IoT technology is discussed in (Karale, 2021).

\subsection{Support}

The need to support millions of devices that belong to different versions from different manufacturers can sometimes be crucial. This is especially true if the system includes older devices that are not supported by the retailer, as well as those that have not been updated to their latest operating platforms.

The challenges that such a picture brings have an impact not only on the operation of the network. In practice, this is perceived as a weakness of the Internet of things technology itself (Atlam, Walters \& Wills, 2018). 


\section{Research Directions}

Like any relatively new technology, the Internet of things offers a wide field for academic and applied research. Although the number of academic publications in the field has grown significantly in recent years, the topics that could be analyzed are practically related to every aspect of the topic.

The presented overview of current research in the field of IoT systematizes some key aspects, including topics such as standardization, system architecture, interoperability and integration, reliability, data storage and processing, scalability, management and self-configuration, performance, identification and identity, energy, and consumption, security and protection of personal data, environmental issues.

In the next section, we will briefly look at two of the most common guidelines for future analysis, namely standardization, and data security and confidentiality.

\subsection{Standardization}

Despite the efforts of the various standardization bodies, there is still no single framework for the concept of the Internet of things. Nevertheless, the contribution of these organizations to the industrial application of the technology, its rapid entry, and overall development is indisputable. The framework of architecture presented above, which is part of the basis of the system, can be taken as an illustration.

Creating uniform standards for such diverse technological standards is a real challenge. Standardization has a key role to play in the future development of the Internet of things. It could affect different aspects of the system. For example, it could have a direct bearing on improving interoperability, but also on the open standards that the Internet uses for things like security, identification, communication. It is believed that standardization will expand the opening of technology to new traders and consumers (Atlam, Walters \& Wills, 2018).

\subsection{Security and privacy}

This challenge forms a key area of future analysis, as, through the continuous transmission of data between different devices and users, it is at the heart of the functioning of the Internet of things. In this sense, the data in its networks are exposed to constant risks, attacks, and threats. Due to low processing power and storage capacity, smart things are vulnerable to attacks because existing security techniques or cryptography are not appropriate (Mohanta et al., 2021). This problem could be considered from different points of view. On one hand, it is a 
prerequisite for gaining public consensus for a more comprehensive application of the Internet of things, and on the other hand, it is associated with numerous technological challenges, which in themselves form topics for analysis. One example of such a study focuses on ensuring the security and confidentiality of data on the Internet of things through the use of mobile computing devices (Liao et al., 2020).

Fig. 4 shows how analysts structure the main categories of data security and confidentiality analysis, namely: key technologies, services, and applications, business applications, social and environmental impact (Colakovi \& Hadžialic, 2018).

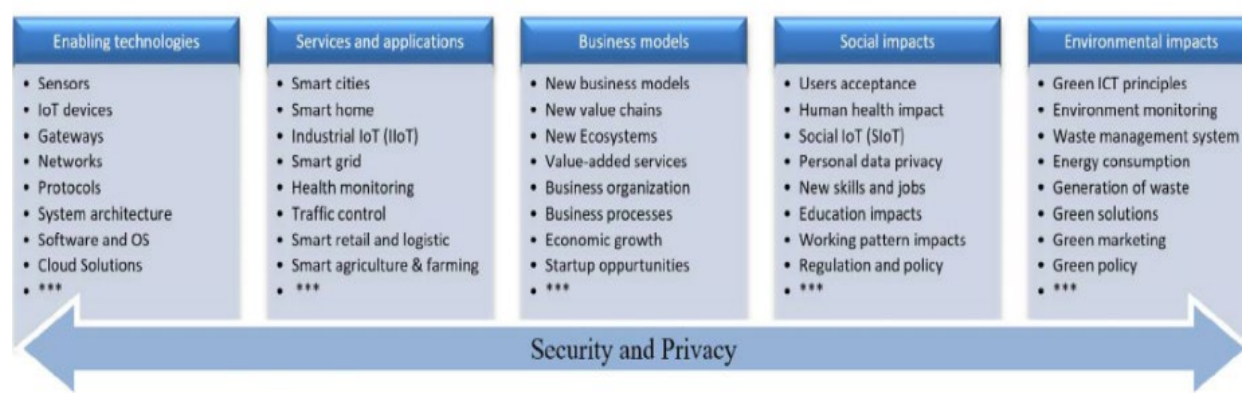

Fig. 4. Categorization of data security and confidentiality analysis

To clarify the complicated security and privacy issues, some authors propose to use the 3-stage protection of pre-working, in-working, and postworking (Yao et al., 2020). In such a way, this physical object-based security architecture allows an analysis of in detail physical objects at different working stages.

\section{Conclusion}

In the last two decades, the Internet of things has gained its place as one of the leading trends in the development of new technologies. The main reasons for this are its potential to improve the quality of human life and its wide public application, which provokes equal interest among the scientific and industrial community. Not coincidentally, technology is described as the next stage in the development of the Internet or as the new industrial revolution. And although there is no generally accepted definition, technology, and standards, the Internet of things is associated with providing the ability to communicate between objects in the physical and cyber world through the Internet. The Internet of things is generally based on the use of different sensors, multi-layered architecture, and a set of communication protocols that vary according to the specifics of the system. 
The Internet of things is used in almost all fields of public and industrial life. Particular importance is attached to environmental monitoring. In industry, its application is often associated with the creation of a qualitatively new type of management of the systems in which it is applied, due to the ability to manage large and different types of data. It is the management of these data and their characteristics that form part of the challenges for the future development of technology.

The IoT is characterized by several features that influence its directions for future development. The review shows that the challenges facing the IoT are mainly related to the standardization of technology, as well as the need to ensure the security and confidentiality of the data that is generated and exchanged. The topics of IoT research cover practically all its aspects, but as leaders, one can distinguish several important areas related to the nature of IoT, namely data collection, data transmission, data processing, and data sharing.

\section{References}

1. Aboubakar, M., Kellilp, M., Rouxp, P.M.: A review of IoT network management: Current status and perspectives. Journal of King Saud University - Computer and Information Sciences, (2021). https://doi.org/10.1016/j.jksuci.2021.03.006.

2. Aggarwal, R., Lal Dasp, M.: RFID Security in the Context of "Internet of Things". In: Proceedings of the First International Conference on Security of Internet of Things, pp. 51-56, New York, NY, USA (2012). https://doi.org/10.1145/2490428.2490435.

3. Ahmad, F. F., Ghenai, Ch., Bettayeb, M.: Maximum power point tracking and photovoltaic energy harvesting for Internet of Things: A comprehensive review. Sustainable Energy Technologies and Assessments 47, 101430 (2021). https://doi.org/10.1016/j.seta.2021.101430.

4. Al Bassam, N., Hussain, Sh. A., Al Qaraghuli, A., Khan, J., Sumesh, E.P., Lavanya, V.: IoT based wearable device to monitor the signs of quarantined remote patients of COVID-19. Informatics in Medicine Unlocked 24, 100588 (2021). https://doi.org/10.1016/j.imu.2021.100588.

5. Arellanes, D., Lau, K.K.: Evaluating IoT service composition mechanisms for the scalability of IoT systems. Future Generation Computer Systems 108, 827-848 (2020). https://doi.org/10.1016/j.future.2020.02.073.

6. Atlam, H., Walters, R., Wills, G.: Internet of Things: State-of-the-Art, Challenges, Applications, and Open Issues. International Journal of Intelligent Computing Research. 9(3), 928-938 (2018).

7. Babun, L., Denney, K., Celik, Z. B., McDaniel, P., Uluagac, A.S.: A survey on IoT platforms: Communication, security, and privacy perspectives. Computer Networks 192, 108040 (2021). https://doi.org/10.1016/j.comnet.2021.108040. 
8. Bahashwan, A.A., Anbar, M., Abdullah, N., Al-Hadhrami, T., Hanshi, S.M.: Review on common IoT communication technologies for both long-range network (LPWAN) and short-range network. In: Saeed F., Al-Hadhrami T., Mohammed F., Mohammed E. (eds) Advances on Smart and Soft Computing. Advances in Intelligent Systems and Computing, vol. 1188 (2021). https://doi.org/10.1007/978-981-15-6048-4_30.

9. Baig, M., Afifi, S., Hosseini, H., Mirza, F.: A Systematic Review of Wearable Sensors and IoT-Based Monitoring Applications for Older Adults - a Focus on Ageing Population and Independent Living. Journal of Medical Systems 43(8), 233 (2018). doi: 10.1007/s10916-019-1365-7.

10. Balan, Z., Ramiah, H., Rajendran, J., Vitee, N., Shasidharan, P. N., Yin, J., Mak, P.I., Martins, R.P.: A coin-battery-powered LDO-Free 2.4-GHz Bluetooth Low Energy/ZigBee receiver consuming $2 \mathrm{~mA}$. Integration 66, 112-118 (2019). https://doi.org/10.1016/j.vlsi.2019.01.009.

11.Bello, O., Zeadally, Sh., Badra, M.: Network layer inter-operation of Device-toDevice communication technologies in Internet of Things (IoT). Ad Hoc Networks 57, 52-62, (2017), https://doi.org/10.1016/j.adhoc.2016.06.010.

12. Borissova, D., Cvetkova, P., Garvanov, I., Garvanova, M.: A Framework of Business Intelligence System for Decision Making in Efficiency Management. In: Saeed K., Dvorsky J. (eds) Computer Information Systems and Industrial Management. CISIM 2020. Lecture Notes in Computer Science, vol. 12133 pp 111-121. Springer, Cham (2020). https://doi.org/10.1007/978-3-030-47679-3_10.

13. Borissova, D., Dimitrova, Z., Dimitrov, V.: How to Support Teams to be Remote and Productive: Group Decision-Making for Distance Collaboration Software Tools. Information and Security. Digital Transformation, Cyber Security and Resilience 46, 36-52 (2020).

14. Borissova, D., Dimitrova, Z., Garvanova, M., Garvanov, I., Cvetkova, P., Dimitrov, V., Pandulis, A.: Two-stage decision-making approach to survey the excessive usage of smart technologies. Problems of Engineering Cybernetics and Robotics 72, 3-16 (2020). https://doi.org/10.7546/PECR.73.20.01.

15. Borissova, D., Mustakerov, I.: A concept of intelligent e-maintenance decision making system. Innovations in Intelligent Systems and Applications, 2013 IEEE International Symposium on. 19-21 June 2013, https://doi.org/10.1109/INISTA.2013.6577668.

16. Boursianis, A.D., Papadopoulou, M.S., Diamantoulakis, P., Liopa-Tsakalidi, A., Barouchas, P., Salahas, G., Karagiannidis, G., Wan, Sh., Goudos, S.K.: Internet of Things (IoT) and Agricultural Unmanned Aerial Vehicles (UAVs) in smart farming: A comprehensive review. Internet of Things, 100187 (2020). https://doi.org/10.1016/j.iot.2020.100187.

17. Brous. P., Janssen, M., Herder, P.: The dual effects of the Internet of Things (IoT): A systematic review of the benefits and risks of IoT adoption by organizations. International Journal of Information Management 51, 101952 (2020). https://doi.org/10.1016/j.ijinfomgt.2019.05.008. 
18. Carlos, A.D.J., Estrada, L.R., Augusto, C.R.C., Patricia, A.C.P., Alberto, P.M.M., Enrique, R.G.R., Cesar, M.O.R., Alfredo, O.G.D., Andres, C.M.C.: Monitoring system of environmental variables for a strawberry crop using IoT tools. Procedia $\begin{array}{llll}\text { Computer } & \text { Science } & 170, & 1083-1089\end{array}$ https://doi.org/10.1016/j.procs.2020.03.067.

19. Chalappuram, A., Sreesh, P.R., George, J.M.: Development of 6LoWPAN in Embedded Wireless System. Procedia Technology 25, 513-519 (2016). https://doi.org/10.1016/i.protcy.2016.08.139.

20.Choi, W., Kim, J., Lee, S.E., Park, E.: Smart home and internet of things: A bibliometric study. Journal of Cleaner Production 301, 126908 (2021). https://doi.org/10.1016/j.jclepro.2021.126908.

21. Cisco (2014) Attaining IoT Value: How to Move from Connecting Things to Capturing Insights. Available from:

https://www.cisco.com/c/dam/global/en_za/solutions/trends/iot/docs/iot-dataanalytics-white-paper.PDF, last accessed 2021/08/03.

22. Cisco (2014) The Internet of Things Reference Model. White Paper. Draft Controlled Distribution. Available from:

http://cdn.iotwf.com/resources/71/IoT_Reference_Model_White_Paper_June 4 2014.pdf, last accessed 2021/08/03.

23. Clement, J., Crutzen, N.: How Local Policy Priorities Set the Smart City Agenda. Technological Forecasting and Social Change 171, 120985 (2021). https://doi.org/10.1016/j.techfore.2021.120985.

24. Colakovi, A., Hadžialic, M.: Internet of Things (IoT): A review of enabling technologies, challenges, and open research issues. Computer Networks 144, 17 39 (2018). https://doi.org/10.1016/j.comnet.2018.07.017.

25. Debauche, O., Mahmoudi, S., Manneback, P., Assila, A.: Fog IoT for Health: A new Architecture for Patients and Elderly Monitoring. Procedia Computer Science 160, 289-297 (2019). https://doi.org/10.1016/j.procs.2019.11.087.

26. DeFeo, Ch.: Chapter 9 - Energy Harvesting and the Internet of Things. Eds: M. Dastbaz, C. Pattinson, B. Akhgar, Green Information Technology - A Sustainable Approach, pp. 151-160 (2015). https://doi.org/10.1016/B978-0-12-8013793.00009-7.

27. Dhanvijay, M.M., Patil, Sh.C.: Internet of Things: A survey of enabling technologies in healthcare and its applications. Computer Networks 153, 113-131 (2019). https://doi.org/10.1016/j.comnet.2019.03.006.

28. Evans, D.: The Internet of Things: How the Next Evolution of the Internet Is Changing Everything. Cisco White Paper. Available from: http://www.cisco.com/web/about/ac79/docs/innov/IoT IBSG 0411FINAL.pdf, last accessed 2021/08/03.

29. Fatkieva, R., Evnevich, E., Yoshinov, R.: State-of-the art and trends in network security control. Problems of Engineering Cybernetics and Robotics 72, 41-55 (2020). https://doi.org/10.7546/PECR.72.20.05. 
30. Fernandex-Carames, T., Fraga-Lamas, P.: A review of human-centered IoTconnected smart labels for the Industry 4.0. IEEE Access 6, 25939-25957 (2018). https://doi.org/10.1109/ACCESS.2018.2833501.

31. Garvanov, I., Garvanova, M., Borissova, D., Vasovic, B., Kanev, D.: Towards IoTBased Transport Development in Smart Cities: Safety and Security Aspects. In: Shishkov B. (eds) Business Modeling and Software Design. BMSD 2021. LNBIP, vol. 422. pp 392-398, (2021). https://doi.org/10.1007/978-3-030-79976-2 27.

32. Gopikumar, S., Banu, J.R., Robinson, Y.H., Shanmuganathan, V., Kadry, S., Rho, S.: Novel framework of GIS based automated monitoring process on environmental biodegradability and risk analysis using Internet of Things. Environmental Research 194, 110621 (2021). https://doi.org/10.1016/j.envres.2020.110621.

33. Guimaraes, C.S.S., de Andrade, M., De Avila, F.R., De Oliveira Gomes, V.E., Nardelli, V.C.: IoT architecture for interoperability and monitoring of industrial nodes. Procedia Manufacturing 52, 313-318 (2020). https://doi.org/10.1016/j.promfg.2020.11.052.

34. Guliashki, V., Marinova, G., Groumpos, P.: Multi-objective optimization approach for energy efficiency in microgrids. IFAC-PapersOnLine 52(25), 477482 (2019).

35. Gustafson, D.H., DuBenske, L.L., Atwood, A.K., Chih, M.Y., Johnson, R.A., McTavish, F., Quanbeck, A., Brown, R.L., Cleary, J.F., Shah, D.: Reducing symptom distress in patients with advanced cancer using an e-alert system for caregivers: pooled analysis of two randomized clinical trials. Journal of Medical Internet Research 19(11), (2017). https://doi.org/10.2196/jmir.7466.

36. Hassani, H., Gringoli, F., Leith, D.J.: Quick \& plenty: Achieving low delay \& high rate in 802.11 ac edge networks. Computer Networks 187, 107820 (2021). https://doi.org/10.1016/j.comnet.2021.107820.

37. Hofer-Schmitz, K., Stojanovic, B.: Towards formal verification of IoT protocols: A review. Computer Networks 174, 107233 (2020). https://doi.org/10.1016/j.comnet.2020.107233.

38.Huang, Ch.H., Chen, P.J., Lin, Y.J., Chen, B.W., Zheng, J.X.: A robot-based intelligent management design for agricultural cyber-physical systems. Computers and Electronics in Agriculture 181, 105967 (2021), https://doi.org/10.1016/j.compag.2020.105967.

39. International Telecommunication Union. (2012) Overview of the Internet of Things. Next Generation Networks. Telecommunication Standardization Sector of ITU. Available from: https://www.itu.int/rec/T-REC-Y.2060-201206-I, last accessed 2021/08/03.

40. John Dian, F., Vahidnia, R., Rahmati, A.: Wearables and the Internet of Things (IoT), Applications, Opportunities, and Challenges: A survey. IEEE Access 8, 69200-69211 (2020), https://doi.org/10.1109/ACCESS.2020.2986329.

41.Karale, A.: The challenges of IoT addressing security, ethics, privacy, and laws. Internet of Things 15, 100420 (2021), https://doi.org/10.1016/j.iot.2021.100420. 
42. Kim, K., Cho, K., Lim, J., Jung, Y.H., Sung, M.S., Kim, S.B., Kim, H.K.: What's your protocol: Vulnerabilities and security threats related to Z-Wave protocol. Pervasive and Mobile Computing 66, 101211 (2020), https://doi.org/10.1016/j.pmcj.2020.101211.

43. Kirchev, L., Blyantov, M., Georgiev, V., Boyanov, K.: A communication model supporting process migration in grid. In: Proc. EXPGRID workshop on large-scale distributed systems of the 15 th international symposium on High-Performance Distributed Computing (HPDC'06), pp. 31-39, Paris, France (2006).

44. Kosmatos, E., Tselikas, N., Boucouvalas, A.: Integrating RFIDs and smart objects into a unified Internet of things architecture. Advances in Internet of Things 1(1), 5-12 (2011), http://dx.doi.org/10.4236/ait.2011.11002.

45. Krasteva, R., Boneva, A., Georchev, V., Stoianov, I.: Application of wireless protocols Bluetooth and ZigBee. in telemetry system development. Problems of Engineering Cybernetics and Robotics 55, 30-38 (2005).

46. Kuang, J., Li, L.: ZigBee-based architecture design of imperceptible smart home system. In: Liu Q., Liu X., Li L., Zhou H., Zhao HH. (eds) Proc. of the 9th Int. Conference on Computer Engineering and Networks. Advances in Intelligent Systems and Computing, vol. 1143, pp. 1003-1011. Springer, Singapore (2020), https://doi.org/10.1007/978-981-15-3753-0_100.

47.Liao, B., Ali, Y., Nazir, S., He, L., Khan, H.U.: Security analysis of IoT devices by using mobile computing: A systematic literature review. IEEE Access 8, 120331-120350 (2020), https://doi.org/10.1109/ACCESS.2020.3006358.

48. Madakam, S., Ramaswamy, R., Tripathi, S.: Internet of Things (IoT): A literature review. Journal of Computer and Communications 3(5). Article ID: 56616 (2015). http://dx.doi.org/10.4236/jcc.2015.35021.

49. Margherita, E.G., Braccini, A.M.: Managing industry 4.0 automation for fair ethical business development: A single case study. Technological Forecasting and Social Change 172, 121048 https://doi.org/10.1016/j.techfore.2021.121048.

(2021).

50. Marinova, G.: Overview on FPGA design implementations for autonomous cars. In: Proc. of the 7th International Conference Computer Science and Communication Engineering \& Information Systems and Security, pp. 41-50. UBT, Pristina, Kosovo (2018).

51. Marques, P., Manfroi, D., Deitos, E., Cegoni, J., Castilhos, R., Rochol, J., Pignaton, E., Kunst, R.: An IoT-based smart cities infrastructure architecture applied to a waste management scenario. Ad Hoc Networks 87, 200-208 (2019). https://doi.org/10.1016/j.adhoc.2018.12.009.

52. Mesmoudi, Y., Lamnaour, M., El Khamlichi, Y., Tahiri, A., Touhafi, A., Braeken, A.: A middleware based on service oriented architecture for heterogeneity issues within the Internet of things (MSOAH-IoT). Journal of King Saud University Computer and Information Sciences 32(10), 1108-1116 (2020). https://doi.org/10.1016/j.jksuci.2018.11.011.

53. Mohanta, B.K., Jena, D., Ramasubbareddy, S., Daneshmand, M., Gandomi, A.H.: Addressing security and privacy issues of IoT using blockchain technology. IEEE 
Internet of Things Journal 8(2), 881-888, (2021), https://doi.org/10.1109/JIOT.2020.3008906.

54. Moysiadis, V., Sarigiannidis, P., Vitsas, V., Khelifi, A.: Smart farming in Europe. $\begin{array}{lllll}\text { Computer } & \text { Science } & \text { Review } & \end{array}$ https://doi.org/10.1016/j.cosrev.2020.100345.

55. Mustakerov, I., Borissova, D.: An intelligent approach for optimum maintenance strategy defining. Innovations in Intelligent Systems and Applications, 2013 IEEE International Symposium on. 19-21 June 2013, https://doi.org/10.1109/INISTA.2013.6577666.

56. Pattar, S., Buyya, R., Venugopal, K.R., Iyengar, S.S., Patnaik, L. M.: Searching for the IoT resources: Fundamentals, requirements, comprehensive review and future directions. IEEE Communications Surveys \& Tutorials 20(3), 2101-2132 (2018). https://doi.org/10.1109/COMST.2018.2825231.

57. Queralta, J.P., Gia, T.N., Zou, Z., Tenhunen, H., Westerlund, T.: Comparative study of LPWAN technologies on unlicensed bands for M2M communication in the IoT: Beyond LoRa and LoRaWAN. Procedia Computer Science 155, 343-350 (2019). https://doi.org/10.1016/j.procs.2019.08.049.

58. Santhosh, N., Srinivsan, M., Ragupathy, K.: Internet of Things (IoT) in smart manufacturing. IOP Conf. Series: Materials Science and Engineering 764, 012025 (2020). Available from: https://iopscience.iop.org/article/10.1088/1757899X/764/1/012025.

59. Shah, A.S., Nasir, H., Fayaz, M., Lajis, A., Shah, A.: A review on energy consumption optimization techniques in IoT based smart building environments. Information 10(3), 108 (2019). https://doi.org/10.3390/info10030108.

60. Sharabov, M., Tsochev, G.: The use of artificial intelligence in Industry 4.0. Problems of Engineering Cybernetics and Robotics 72, 17-29 (2020). https://doi.org/10.7546/PECR.73.20.02.

61. Sikimic, M., Amovic, M., Vujovic, V., Suknovic, B., Manjak, D.: An overview of wireless technologies for IoT network. In: 19th International Symposium INFOTEH-JAHORINA (INFOTEH), pp. 1-6, (2020), https://doi.org/10.1109/INFOTEH48170.2020.9066337.

62. Simo, A., Dzitac, S., Dzitac, I., Frigura-Iliasa, M., Frigura-Iliasa, F.M.: Air quality assessment system based on self-driven drone and LoRaWAN network. Computer Communications 175, 13-24 https://doi.org/10.1016/j.comcom.2021.04.032.

(2021).

63. Singh, D., Dahiya, M., Kumar, R., Nanda, Ch.: Sensors and systems for air quality assessment monitoring and management: A review. Journal of Environmental Management 289, 112510 (2021). https://doi.org/10.1016/j.jenvman.2021.112510

64. Sokullu, R., Akkas, M.A., Demir, E.: IoT supported smart home for the elderly. Internet of Things 11, 100239 (2020). https://doi.org/10.1016/j.iot.2020.100239.

65. Suresh, P., Daniel, J.V., Parthasarathy, V., Aswathy, R.H.: A state of the art review on the Internet of Things (IoT) history, technology and fields of deployment. In: Proc. of International Conference on Science Engineering and Management 
Research (ICSEMR), pp. 1-8, Chennai, India (2014). https://doi.org/10.1109/ICSEMR.2014.7043637.

66. Talavera, J.M., Tobon, L.E., Gomez, J.A., Culman, M.A., Aranda, J.M., Parra, D.T., Quiroz, L.A., Hoyos, A., Garreta, L.E.: Review of IoT applications in agroindustrial and environmental fields. Computers and Electronics in Agriculture 142(A), 283-297 (2017). https://doi.org/10.1016/j.compag.2017.09.015.

67. Taylor, T.: 6 Most Commonly Used IoT Communication Protocols. TechGenis, (2019). Available from: http://techgenix.com/iot-communication-protocols/, last accessed 2021/08/03.

68. Tianxing, Ma., Yoshinov, R., Osipov, V., Zhukova, N., Schukina, M., Evnevich, E.: Problems of human secure interaction with the internet space. Problems of Engineering Cybernetics and Robotics 75, 15-34 (2021). https://doi.org/10.7546/PECR.75.21.03.

69. Todtenberg, N., Kraemer, R.L A survey on Bluetooth multi-hop networks. Ad Hoc Networks 93, 101922 (2019). https://doi.org/10.1016/j.adhoc.2019.101922.

70. Tournier, J., Lesueur, F., Le Mouel, F., Guyon, L., Ben-Hassine, H.: A survey of IoT protocols and their security issues through the lens of a generic IoT stack. Internet of Things, 100264 (2020). https://doi.org/10.1016/j.iot.2020.100264.

71. Trevathan, J., Schmidtke, S., Read, W., Sharp, T., Sattar, A.: An IoT generalpurpose sensor board for enabling remote aquatic environmental monitoring. Internet of Things 16, 100429 (2021). https://doi.org/10.1016/j.iot.2021.100429.

72. Tsochev, G.R., Yoshinov, R.D., Iliev, O.P.: Key Problems of the critical information infrastructure through Scada Systems Research. SPIIRAS $\begin{array}{lll}\text { Proceedings } & 18(6), & \text { 1333-1356 }\end{array}$ https://doi.org/10.15622/sp.2019.18.6.1333-1356.

73. Vahdat-Nejad, H., Abbasi-Moud, Z., Eslami, S.A., Mansoor, W.: Survey on Context-Aware Healthcare Systems. 2021 IEEE 11th Annual Computing and Communication Workshop and Conference (CCWC), pp. 1190-1196, NV, USA (2021). https://doi.org/10.1109/CCWC51732.2021.9376138.

74. Vodyaho, A., Yoshinov, R., Zhukova, N., Thaw, A.M., Ahmed, A.S.: Fog oriented model for data collection in the networks of mobile devices. In: Proc. of IEEE 10th Int. Conf. on Intelligent Systems, pp. 421-425, Varna, Bulgaria (2020). https://doi.org/10.1109/IS48319.2020.9200138.

75. Yao, X., Farha, F., Li, R., Psychoula, I., Chen, L., Ning, H.: Security and privacy issues of physical objects in the IoT: Challenges and opportunities. Digital Communications and Networks, (2020). https://doi.org/10.1016/j.dcan.2020.09.001.

76. Yugha, R., Chithra, S.: A survey on technologies and security protocols: Reference for future generation IoT. Journal of Network and Computer Applications 169, 102763 (2020). https://doi.org/10.1016/j.jnca.2020.102763. 